\title{
Enabling Machine Learning-Ready HPC Ensembles with Merlin
}

\begin{abstract}
J. Luc Peterson*, Ben Bay, Joe Koning, Peter Robinson, Jessica Semler, Jeremy White, Rushil Anirudh, Kevin Athey, Peer-Timo Bremer, Francesco Di Natale, David Fox, Jim A. Gaffney, Sam A. Jacobs, Bhavya Kailkhura, Bogdan Kustowski, Steven Langer, Brian Spears, Jayaraman Thiagarajan, Brian Van Essen, Jae-Seung Yeom
\end{abstract}

Lawrence Livermore National Laboratory, Livermore, California 94550, USA

\begin{abstract}
With the growing complexity of computational and experimental facilities, many scientific researchers are turning to machine learning (ML) techniques to analyze large scale ensemble data. With complexities such as multi-component workflows, heterogeneous machine architectures, parallel file systems, and batch scheduling, care must be taken to facilitate this analysis in a high performance computing (HPC) environment. In this paper, we present Merlin, a workflow framework to enable large ML-friendly ensembles of scientific HPC simulations. By augmenting traditional HPC with distributed compute technologies, Merlin aims to lower the barrier for scientific subject matter experts to incorporate $\mathrm{ML}$ into their analysis. In addition to its design, we describe some example applications that Merlin has enabled on leadership-class HPC resources, such as the ML-augmented optimization of nuclear fusion experiments and the calibration of infectious disease models to study the progression of and possible mitigation strategies for COVID-19.
\end{abstract}

\section{Introduction}

\subsection{The Problem: Using Machine Learning to Bridge Simulation and Experi- mentation}

A fundamental tenet of scientific investigation is the challenging of models with experimentation. A process that began as a search for analytic descriptions of reality has evolved into the validation of complex computational calculations with increasingly complicated experimental observations. Large scale experiments, such as those performed at the National Ignition Facility (NIF) [1], can produce upwards of a gigabyte of multi-component information on each shot.

\footnotetext{
* Corresponding author

Email address: peterson76@llnl.gov (J. Luc Peterson)
} 
Furthermore, modern high performance computing (HPC) platforms have made it easier than ever to produce large quantities of simulated data. For example, a single instance of a moderately-resolved radiation hydrodynamic simulation of a NIF experiment can produce of order 10 gigabytes of simulation data in six hours on a single Intel Haswell node [2.

In addition to being able to produce large quantities of data from a single simulation and motivated in part by the emergence of scientific machine learning (ML), the creation of large-scale scientific simulation databases is an emerging trend in HPC. Simulations can provide multivariate data (e.g. timeseries, scalar, vector, and image) that are physically correlated by design. Due to their complexity and scale, large simulation datasets with large numbers of samples/instances are an attractive challenge to ML systems. Of the publicly available datasets in the UCI ML repository [3], the largest sample size consists of 63 million instances of time-series medical sensor data [4. Of the 49 physical science datasets, the largest consists of roughly 11 million particle physics simulations 5 intended for scalar classification. These datasets mainly contain scalar numbers (either static or as time-series), so they are gigabytes in size.

Ensemble simulations that produce data on a grid or generate synthetic images may produce much larger numbers of samples and much larger datasets. An ensemble of 10,240 simulations of Earth's weather was run on the K Computer [6. This ensemble produced $1.21 \mathrm{~TB}$ of data and roughly 3.3 million grid slices. A grid slice can be thought of as a 40,960 "pixel" image (i.e. one pixel per grid zone). As a benchmark for HPC-based simulations of multivariate data (scalar, vector, image), the authors of [2] used an on-node producer-consumer task queue system [7] to create a 70 TB database of 60,000 multiphysics simulations. The dataset includes roughly a billion compressed synthetic x-ray images as well as time series and scalars.

As our capacity to produce scientific data has increased, our ability to analyze it has largely failed to keep up. A typical comparison between a NIF experiment and simulation involves the distillation of all data into around 10 scalar quantities, a process that leaves most of the data unanalyzed and the models potentially under-constrained.

One hope is that modern data analytics and machine learning will provide the tools to help scientific analysis keep up with scientific data production. Possible applications include performing near real-time feature selection for data compression or creating surrogate models for expensive simulation codes. Surrogate models could be used to perform sensitivity studies, optimization, uncertainty quantification, and validation of computer models against experiments without running additional HPC simulations.

ML systems require many examples (samples) to build accurate surrogate models, but HPC systems are designed to execute at most only a few concurrent instances of very complex models (e.g. large MPI jobs). This underlying tension between ML requiring many simulations but HPC being optimized for a few means that the creation of HPC simulation datasets used to train ML models must be accomplished with care. Standard HPC workflow toolkits may not be the most efficient way to produce the large ensembles of simulation data required 
for ML model training.

In this paper, we detail the development of a simulation workflow framework, Merlin, whose purpose is to facilitate the creation of large scale ensembles of simulation data suitable for analysis by machine learning tools.

\subsection{Challenges for $M L$ Ensembles in $H P C$}

As a motivating example for the application of ML to an HPC environment, consider a simple "intelligent" sampling workflow, which uses active learning to dynamically select new simulations to add to an ensemble. Since HPC simulations are expensive, it is reasonable to only run simulations if necessary to improve the accuracy of the trained model. The simplest loop to accomplish this involves running simulations, post-processing the raw data from those simulations into salient features, training an ML system on those features, evaluating the error on that training and adding new simulations to reduce the model error.

A major challenge of even this simple workflow is that it is inherently heterogeneous, involving multiple simulation codes (at the least, a simulator and a learner). The simulator must calculate the raw quantities of interest and write them in a data format that can be read efficiently by the learner. However, the simulator and learner are likely optimized for a different set of execution parameters. For instance, learning can be extremely efficient on a graphics processing unit (GPU), but simulating might not be, depending on the application. If the raw data produced by the simulator need to be post-processed prior to ingestion by the learner, such a post-processor would likely have different requirements. This heterogeneous workflow must be coordinated and orchestrated in an efficient manner, potentially across multiple batch allocations and hardware systems, which may or may not be able to directly access each other.

Modern HPC systems were designed and optimized for the efficient execution of a few large scale simulations, instead of the large number of smaller scale simulations necessary to train accurate ML models. Batch scheduling systems are generally not designed to launch thousands to millions of simulations. Parallel file systems can lose performance when presented with large numbers of concurrent reads and writes that overwhelm meta-data servers. Dynamically loaded shared objects can present similar problems.

Fundamentally, creating ML-ready ensembles of HPC simulation data necessitates a workflow technology that can efficiently coordinate asynchronous heterogeneous simulation tasks at scales well beyond the designed operation of HPC systems. Merlin aims to fill this space.

\subsection{Related Existing Workflow Technologies}

Workflow technology is a rich field, where many approaches provide similar functionality. Their underlying mechanisms, implementations, and flexibility vary widely. These technologies range from full workflow tools to scripting languages for procedurally generating tasks. In this section, we briefly survey some existing workflow technologies that have been applied to scientific computing. 
Pegasus [8] and Fireworks [9] are full workflow systems which provide task tracking, generalized scheduling, and Python APIs for creating workflows programmatically. Both allow tasks to be defined using directed acyclic graphs (DAGs). The Fireworks "Launchpad" (built on MongoDB) is a staging mechanism which pushes bundles of work to compute nodes. Pegasus makes use of HTCondor through DAGMan and follows a pull model.

The Sandia Analysis Workbench (SAW) provides users a GUI interface and plugin system using the Eclipse IDE and their integrated OSGi plugin system [10. SAW provides flexibility by implementing plugins as generalized extension points representing abstract configurations that define an interface. Plugins can be tied together to build larger workflow processes, in particular workflows geared towards verification and validation testing $(\mathrm{V} \& \mathrm{~V})$. All components implement a backend Java interface to their workflow engine, allowing components to be used to create an explicit workflow graph and enables task scheduling and monitoring.

The UQ Pipeline [1] has application specific capabilities such as performing sensitivity studies, estimating parameter values, and a host of other features. The UQ Pipeline makes use of CRAM [12] to consolidate jobs in a batch system so that they can achieve high sample counts in their workflows without submitting huge numbers of jobs to HPC clusters.

On the other end of the spectrum, Swift 13 is a popular parallel scripting language. It supports most of the structures expected from a programming language and can create dynamic workflows. Like Pegasus, it follows a data flow model. Swift follows a functional approach, where functions take in data inputs and produce data to be passed to other functions.

While these technologies provide a robust set of features, they are not without their challenges. In broad terms, these workflow technologies fall under two categories: distributed lightweight computing or localized large scale HPC.

Distributed workflow systems tend to have larger sets of features, but provide domain scientists, who are used to working in HPC environments, with an expensive upfront training cost, and can potentially lock scientists into release cycles out of their control. The lack of control on new features can be limiting, especially as new forms of compute hardware become available (GPUs, ASICs, etc.) and when testing on leadership class machines. Security imposes additional constraints in HPC environments, and retaining system security and integrity often disqualifies commercial tools due to the necessary efforts to verify software before use. Other limitations include the necessary traceability of user actions which disqualifies services that obfuscate such information. More fully fledged workflow tools like Pegasus and Fireworks are attractive, but obtaining the security approvals to stand up their backend technologies in HPC environments can prove cumbersome.

HPC-focused workflow technologies, such as the UQ Pipeline, Swift, CONDOR 14] and EMEWS [15] (the latter two built upon Swift), focus on executing many tasks in a single large MPI job running on a single machine. Approaches like Swift that avoid coordination via the filesystem show advantages in scaling, but their focus on single batch job ensembles precludes cross- system workflow 
coordination. On the other hand, Maestro [16] can coordinate across batch jobs, but does so via filesystem coordination and live background processes running on login nodes, limiting throughput. Programmatic interfaces, such as those in the UQ Pipeline and Swift, and GUI-interfaces like in SAW, represent barriers for user adoption, since their interfaces represent a new language for subject matter experts to learn. This contrasts with Maestro's YAML specification for DAG definition, which has users define workflow steps in shell syntax.

\subsection{Summary of Merlin and Paper Outline}

In this paper we explore the creation of a lightweight, scalable workflow suite specifically designed to enable large ensembles of HPC simulations tailored for easy analysis by machine learning tools. The software, Merlin, combines work-

flow and HPC technologies in a framework that enables the scalable execution of heterogeneous workflows suitable for next-generation ML-driven workflows. It is compatible with present day HPC systems, security models and front-line multiphysics codes. Sec. 2 details the design of Merlin, while Sec. 3 demonstrates its use on a variety of applications. In particular we show that Merlin can handle a variety of ensemble types, from the efficient execution of many lightweight tasks, to multi-machine heterogeneous simulator-learner optimization workflows to large multiphysics-based ensembles. We demonstrate Merlin's capability by reporting on the creation of an unprecedentedly large inertial confinement fusion (ICF) dataset, consisting of approximately 100 million simulations and 4.8 billion images, that was generated on the Sierra Supercomputer at Lawrence Livermore National Laboratory. Finally, we show that Merlin's ability to apply surge computing resources to complicated dynamic workflows helped enable the quick re-calibration of an existing infectious disease model to study the progression of and possible mitigation strategies for COVID-19.

\section{Merlin}

An ML-integrated workflow system designed to create massive ensembles of multi-physics simulations has a number of design considerations that are not present in smaller workflows. In particular, such a system must be able to:

- Operate in an HPC environment

- Produce and process the data from $>10^{5}$ MPI-driven simulations

- Support both in-situ (on-node) and in-transit (concurrent) analysis

- Support multi-machine and multi-batch slot workflows

- Support multiple executable types

With these considerations as motivation, we next discuss the technological choices that went into Merlin and perform a series of tests on its performance. 


\subsection{Design Considerations}

The primary design consideration for Merlin is the environment in which large scale simulations must be run, namely large HPC systems such as Sierra. These systems operate at the leading edge of computational technology and at a uniquely large scale, under constrained access models. Efficiently executing a single instance of a simulation at these scales can require customized libraries, compilers, environment setup, and control. They also require effective interfacing with a system batch scheduler, which may, along with the operating system on the machine, be unique. At these large scales, failures of hardware and software are to be expected.

These constraints alone imply that a "one size fits all" approach to a workflow is likely insufficient. Put another way, subject matter experts need to be able to have fine and programmatic control over the execution of their component applications. Individual components may need to run on different machines, each with their own batch scheduling systems and potentially their own isolated file systems. Furthermore, the competition for available computational resources on these machines can be fierce, with simulation throughput highly dependent on the background load on the system.

Beyond the unique operating environment of heterogeneous state-of-the-art HPC systems, ensembles of multi-physics simulations will likely need to consist of a large quantity of individual samples. As an example, the machine learning model used in 2] was trained on an ensemble of roughly 60,000 ICF simulations. Since ML-systems require a large number of individual sample points to obtain an accurate representation of the response surface, a suitable workflow needs to be able to accommodate and coordinate the execution of at least tens of thousands of MPI-based simulations.

Another consideration is that these multi-physics codes can produce a large quantity of raw data, for instance mesh-based fields such as temperature and density. Prior to being fed into a machine learning model, these raw quantities must be post-processed into derived quantities, such as synthetic diagnostic signatures, or hand-curated features. While the raw data itself can be quite large, of order gigabytes or more per simulation, the derived quantities themselves can be of order megabytes, depending on the type of data of interest. This necessity to post-process implies that an ML-driven workflow needs to be able to handle both in-situ (processed in-line by the parent simulation code) and in-transit (processed by a separate data processing step) analysis.

Additionally, an ML-driven large scale HPC ensemble may need to be executed on multiple machines across multiple batch slots. The need for multiple batch slots is straightforward - many leadership class machines have time limits to the length of a single job. Unless all simulations in an ensemble can complete on the allocated resources prior to the time limit, the workflow will need to be able to coordinate and spill-over into multiple resource allocations. These batch slots, however, might not be on the same machine. For example, the ML systems might train most efficiently on different hardware than is optimal for the main simulation code. Such an ensemble would consist of different executables, whose work needs to be coordinated. 
All of these considerations motivate a series of technological choices to more easily enable ML-suitable large ensembles. Scalability arguments suggest that simulation coordination needs to be accomplished via message passing, instead of queries to files or the batch system. The need for multiple machines and batch systems implies that workflow coordination should persist outside of any particular job. The unique HPC environments and the customization required to effectively execute multiphysics codes on leadership class machines means that the workflow interface needs to accommodate the shell-based commands subject matter experts require for their parallel jobs. Data formats should be portable across machines and languages, with the capability for in-memory and on-disk inter-operability. ML-driven ensembles may require distributed and dynamic task creation to avoid bottlenecks at scale and to enable intelligent sampling schemes.

\subsection{System Design}

We describe in this section Merlin, its underlying software components, its user-facing interface, and its task-generation algorithm. The primary requirements set out in the previous section are scalability, flexibility, and ease of use.

To enable cross-machine and dynamic task creation, Merlin is built upon a producer-consumer workflow model. As an underlying library, we choose Celery [17] for both its asynchronous task scheduling and its ability to rely on multiple task brokers and results backend. While Merlin can be configured for any of Celery's supported brokers and backends, defaults are RabbitMQ [18] and Redis 19 for their scalability and reliability.

As an interface to Celery, we leverage Maestro [16. Maestro has a shell-like interface for defining workflows. A user defines a "study" with distinct "steps" of different "parameters" and dependencies. The dependencies and parameters define a directed acyclic graph (or DAG). Each step has a command section written in an arbitrary shell languag $\oint^{1}$ with variable key words for parameters and DAG-workspaces. At runtime, the DAG gets expanded with the full set of parameters replacing the key words and the dependency chains completed. Merlin takes these commands and dependencies and translates them into Celery task constructions of subprocess commands, which are executed by workers receiving the task in a directory unique to that task.

The shell-like interface allows flexibility in both workflow component syntax (for instance to call MPI-driven components through flux [20, to leverage libraries like Conduit 21] for data exchange and translation or to execute components in containerized environments). When combined with the DAG meta-construct and a producer-consumer model, a wide variety of workflow topologies are supported. For instance, workflow steps can make additional

\footnotetext{
${ }^{1}$ We extend Maestro's original syntax by setting the shell to be a user-defined parameter that can vary with the step. This allows for steps to be written not only in different shells (like bash or tcsh), but also more complete languages, such as python or lua. In addition to this step-wise syntax customization, the use of an artibrary shell facilitates composite multi-language workflows.
} 


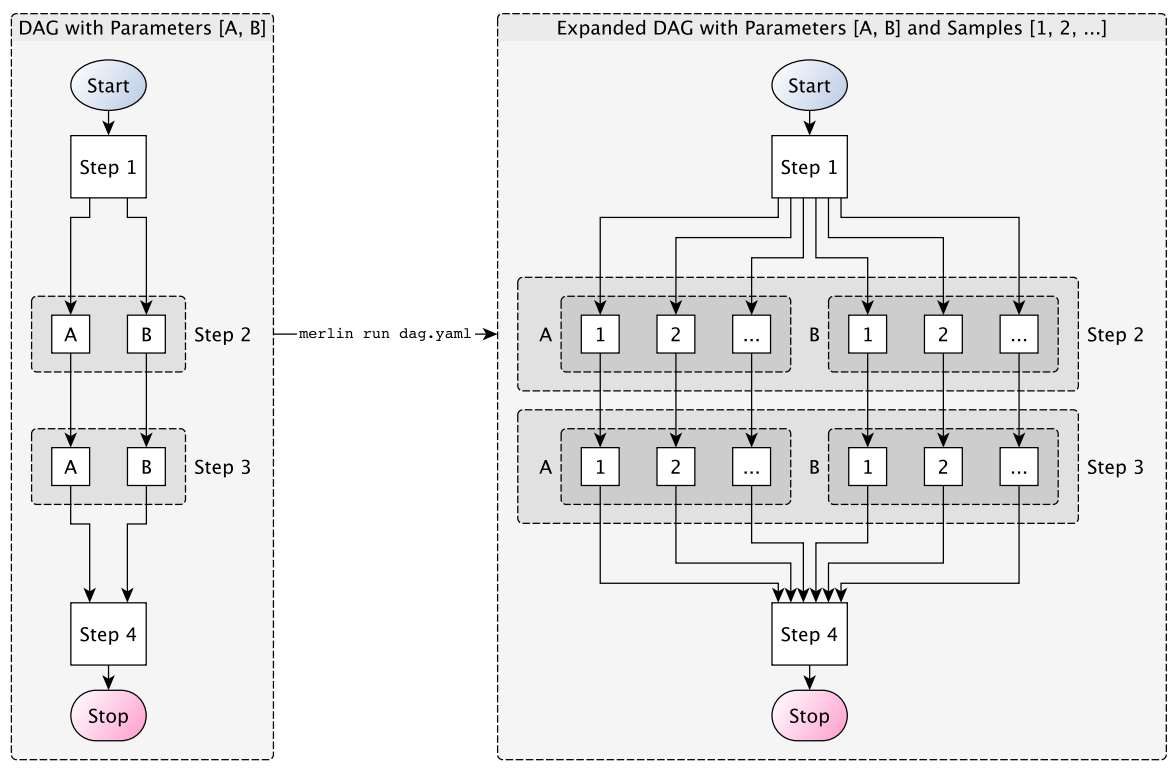

Figure 1: Merlin takes a compact directed acyclic graph (DAG) with discrete parameter values $(\mathrm{A}, \mathrm{B}, \ldots)$ and dependencies and expands it to include hierarchically and dynamically generated samples $(1,2, \ldots)$, which are layered onto the parameter set. Samples have a known topological dependence, which can be exploited for performance, whereas DAG dependencies can be more complicated and require more time to calculate. A layered approach allows for both flexibility and scalability.

calls to Merlin to enqueue other workflow studies, or can use language syntax to implement dynamic flow control and branching. Merlin's use of Maestro as an interface to Celery allows for workflow flexibility in a programmable and HPC-intuitive syntax.

For scalability, we augment Maestro's notion of workflow "parameters" with "samples." Samples are variable sets that are executed for each distinct parameter combination (see Figure 1). This makes their workflow topology simple (they are embarrassingly parallel for a given parameter combination), a fact that we exploit for scalable task generation and execution.

In particular, Merlin takes advantage of the producer-consumer workflow model inherent to Celery to construct a hierarchical task generation algorithm. At producer runtime (via the command merlin run), the algorithm parses the total number of samples to be executed and breaks them into a hierarchical grouping of multiple levels. Instead of enqueuing the entire ensemble of samples at once, Merlin enqueues only a single task at the top of the hierarchy containing the metadata necessary to enqueue its child tasks. The process recursively executes down the hierarchy until reaching the bottom nodes, at which point the actual simulations are enqueued. As consumers (merlin run-workers) come online, they begin to dynamically populate the task queue, in addition to 
beginning to work on actual simulations.

As an additional design feature, we exploit Celery's ability to set tasking priority and explictly prioritize simulation tasks over task-creation tasks. In testing, this significantly improved server stability for very large ensembles by reducing the hardware constraints on the server. Without such a feature it's possible for one user to enqueue a large number of tasks, effectively reserving space on the server for their workflow without consuming those tasks. This problem becomes particularly acute for real-world simulations with long execution times: task-creation is fast but task-consumption is slow, so task-creation quickly outpaces task-consumption and strains the server. However, by telling workers to prioritze draining the queue over filling the queue, we guard against this pathology.

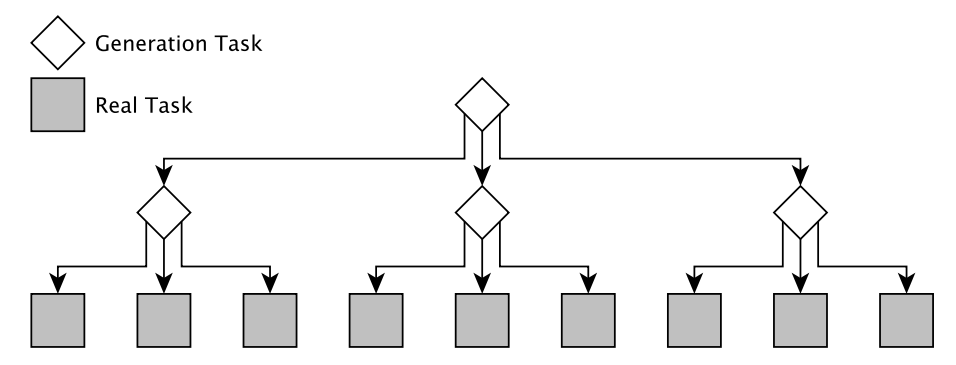

Figure 2: Total tasks in a simple hierarchy of 9 real tasks, divided with up to 3 tasks per level. Generation tasks (white diamonds) dynamically generate child tasks and populate the queue server. True workflow steps are executed in real tasks (gray squares).

As an example, consider using 4 workers to execute the workflow displayed in Fig. 2. It has 9 samples ("real tasks") defined in a hierarchy of up to 3 branches at each level (for a total of 3 levels: a single node at the top, 3 in the middle layer and 9 on the bottom). Upon execution, a single task is enqueued and executed by the first worker, which creates 3 more tasks (the middle layer). Each of these are executed asynchronously by the other available 3 workers, which each create 3 tasks, the bottom of the tree, comprised of the actual simulations. As soon as the first simulation task in enqueued, the original first worker, having finished its generation tasks and now sitting idle, sees the new task and begins executing the first simulation. Meanwhile the other workers finish creating the other 8 simulation tasks. As each worker finishes its generation tasks, it can start working on a real task. In this fashion, the task queue gets dynamically populated and drained.

The task creation hierarchy provides a few benefits. Firstly it means that task enqueuing is an efficient process, since merlin run needs only to populate the queue server with the metadata required to create the tasks, not the tasks themselves. Secondly, it makes task creation a scalable process itself, since the load is spread out dynamically among workers. Finally, it means that actual simulation work can begin executing in a shorted wallclock time, as soon as 
the first simulation task is enqueued, instead of having to wait for the entire simulation ensemble to be created.

\subsection{Performance Analysis}

In this section, we perform a series of tests to measure the performance of Merlin on an idealized but simple workflow of independent tasks each consisting of the shell command sleep 1, which represents a null simulation. To create unique samples the workflow step also includes a comment with the sample identification. The goal is to measure the overhead associated with using Merlin to run simulations. We choose 1 second because real world HPC simulations are unlikely to complete more quickly and a sleep time of 0 seconds could allow system level jitter to confuse results.

All of these tests were performed on the Pascal supercomputer at Lawrence Livermore National Laboratory, with access to a standalone in-house RabbitMQ and Redis server, residing on a single node of an adjacent machine in the same compute center and accessible by all compute nodes of Pascal.

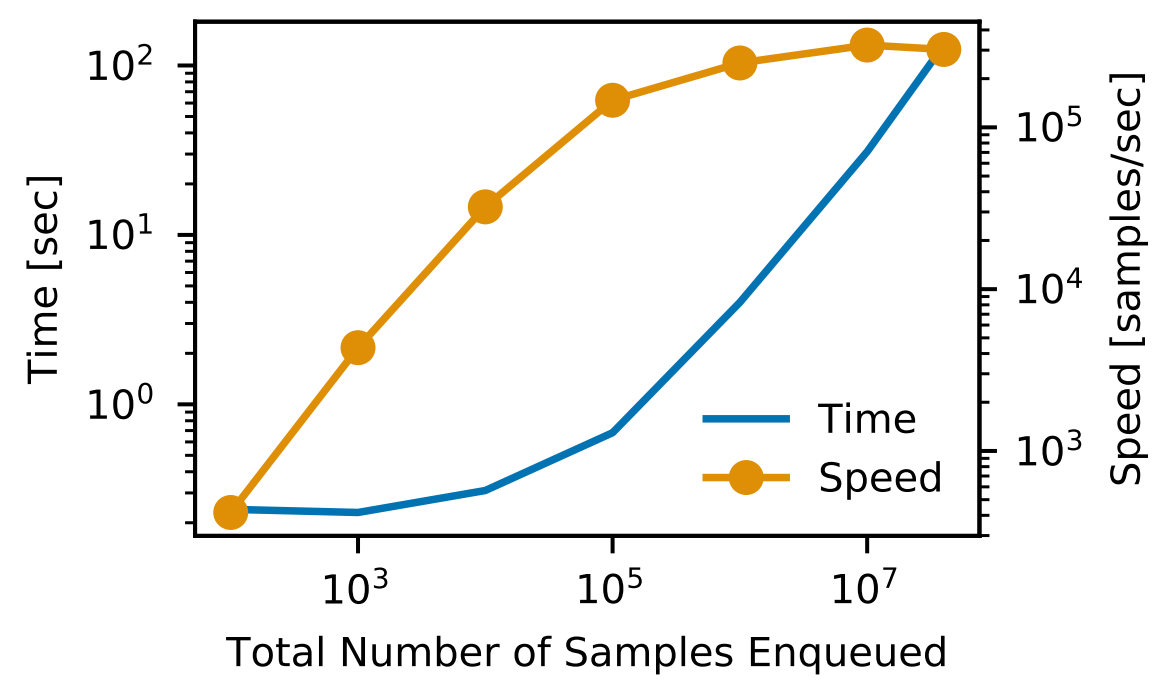

Figure 3: Scalability of task enqueuing time [seconds] and speed [samples/second] with total number of samples.

The first test times how long it takes to enqueue studies of different sizes. Figure 3 measures the time it takes merlin run to create task hierarchy metadata and populate the queue server, for simulation ensembles of various sizes. Both the total time required to populate the server as well as the enqueuing speed (defined by the ratio of the total time to the total number of samples) are plotted for studies ranging from 100 samples up to 40 million samples. Above 40 million, the RabbitMQ server hit a system-defined limit on allowable message sizes $(2.1 \mathrm{~GB})$. The task enqueuing reaches a peak speed around $3 \times 10^{5}$ samples 
per second and plateaus at ensemble sizes greater than $10^{5}$. At smaller ensembles task queuing speed is lower due to the overhead of creating the ensemble data structure dominating the process. Nonetheless, ensembles at this scale can be queued in less than 1 second, and 40 million simulations take around 100 seconds to queue.

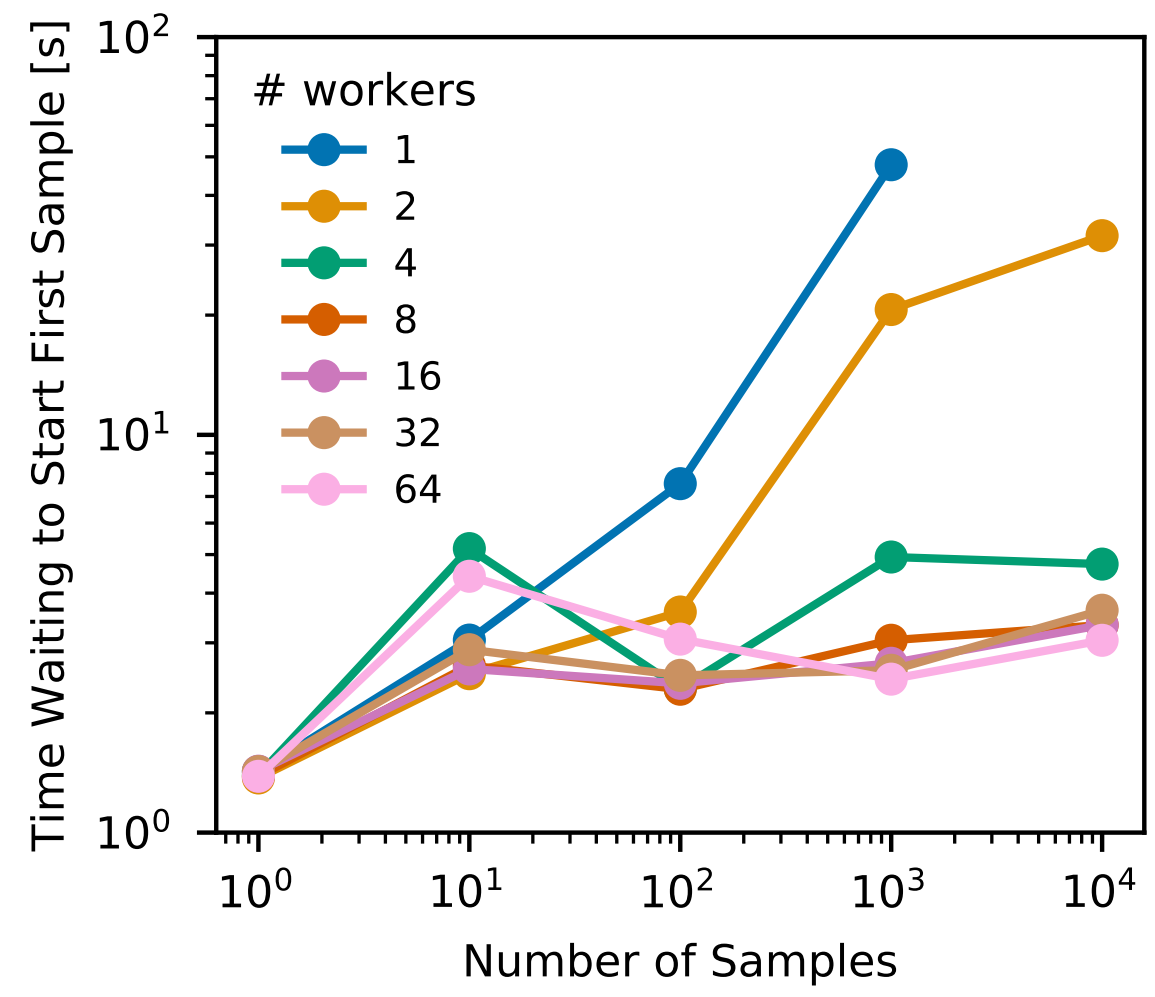

Figure 4: Pre-sample startup time. Number of seconds taken by a workflow between the activation of workers and the starting of sample processing.

Figure 4 measures how long workers wait before executing the first simulation. This represents the time spent unpacking the task hierarchy before simulations begin. Minimizing this time means that batch allocations and their dedicated resources do not lie idle during ensemble initialization. As ensemble size increases, the time spent waiting to start simulations increases as well; however, adding extra worker threads causes this time to drop, for instance allowing a 1000-sample ensemble to begin in 3 seconds with 4 worker threads as opposed to 50 seconds with only 1 thread. Adding additional workers doesn't appreciably lower the time, since there are enough workers to unpack down to the first simulation leaf of the hierarchy.

Together, Figures 3 and 4 demonstrate that the task hierarchy generation 
algorithm reduces the overhead associated with creating simulation ensembles. They make Merlin an effective producer-consumer workflow model with a nearly non-blocking producer stage.

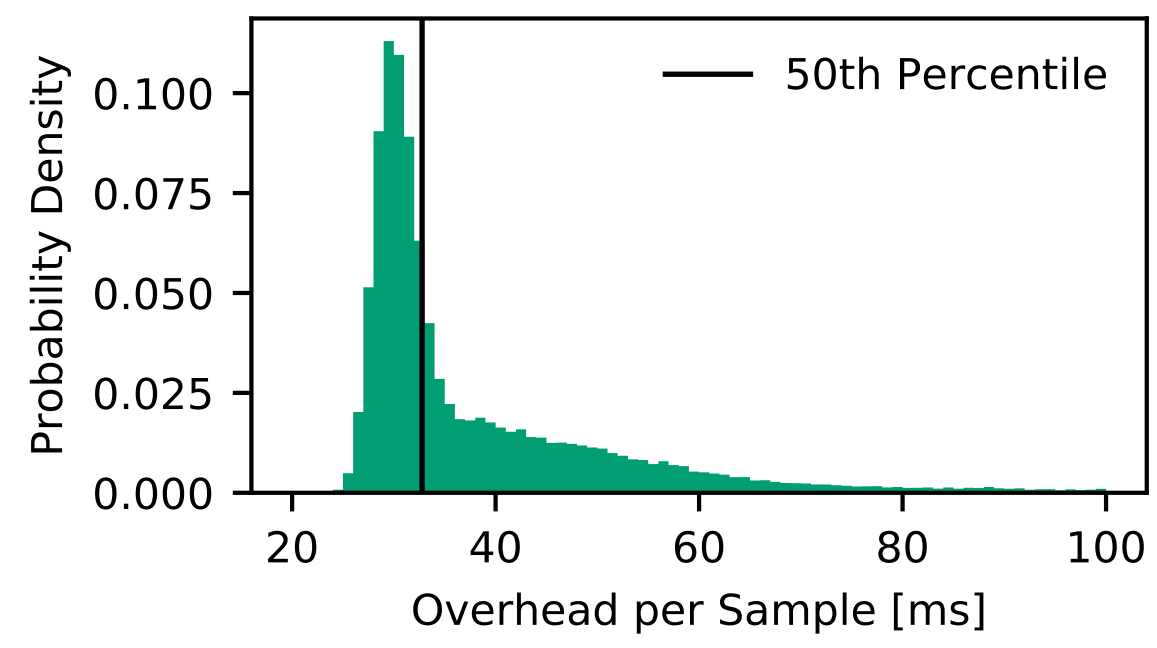

Figure 5: Histogram of all individual task times for approximately 900,000 tasks spawned by the 48 workflows comprising this overhead study. Not all tasks are equal; in the hierarchical task creation algorithm, there are different classes of tasks (task-creation, generic workflow steps). This heterogeneity plus system jitter likely result in the right-skewed distribution. Observations with a modified z-score greater than 5 are classified as outliers and excluded from the plot. The bulk of system tasks are small and take less than 50 milliseconds.

Another measure of overhead is the time spent by the workflow system not running the simulation itself. Figure 5 shows a histogram of this overhead for all roughly 900,000 simulations run in the studies in Fig. 4, as defined as the time between when a worker acknowledges receiving a task and when it tells the central RabbitMQ server it has finished, minus the 1-second sleep interval. This time includes the time needed to create a unique directory in which to execute the sample, create the script that contains the sample-unique instructions and any network-related traffic. Average overhead is tens of milliseconds per sample, with a median value of $32.8 \mathrm{~ms}$. The mode peaks slightly below that, but a long tail extends out towards $100 \mathrm{~ms}$. Nonetheless, the overhead associated with each sample is small compared to the expected compute time for an HPC-based simulation.

Figure 6 shows the total time required to execute all of the simulations as a function of the number of available workers (solid with circles) alongside dashed curves of ideal scaling, which correspond to each worker taking exactly 1 second to execute the null simulation. In other words, we expect $10^{3}$ samples to take $10^{3}$ seconds with one worker thread but half the time with two workers. As the number of samples increases, the data trend towards the ideal scaling, showing 


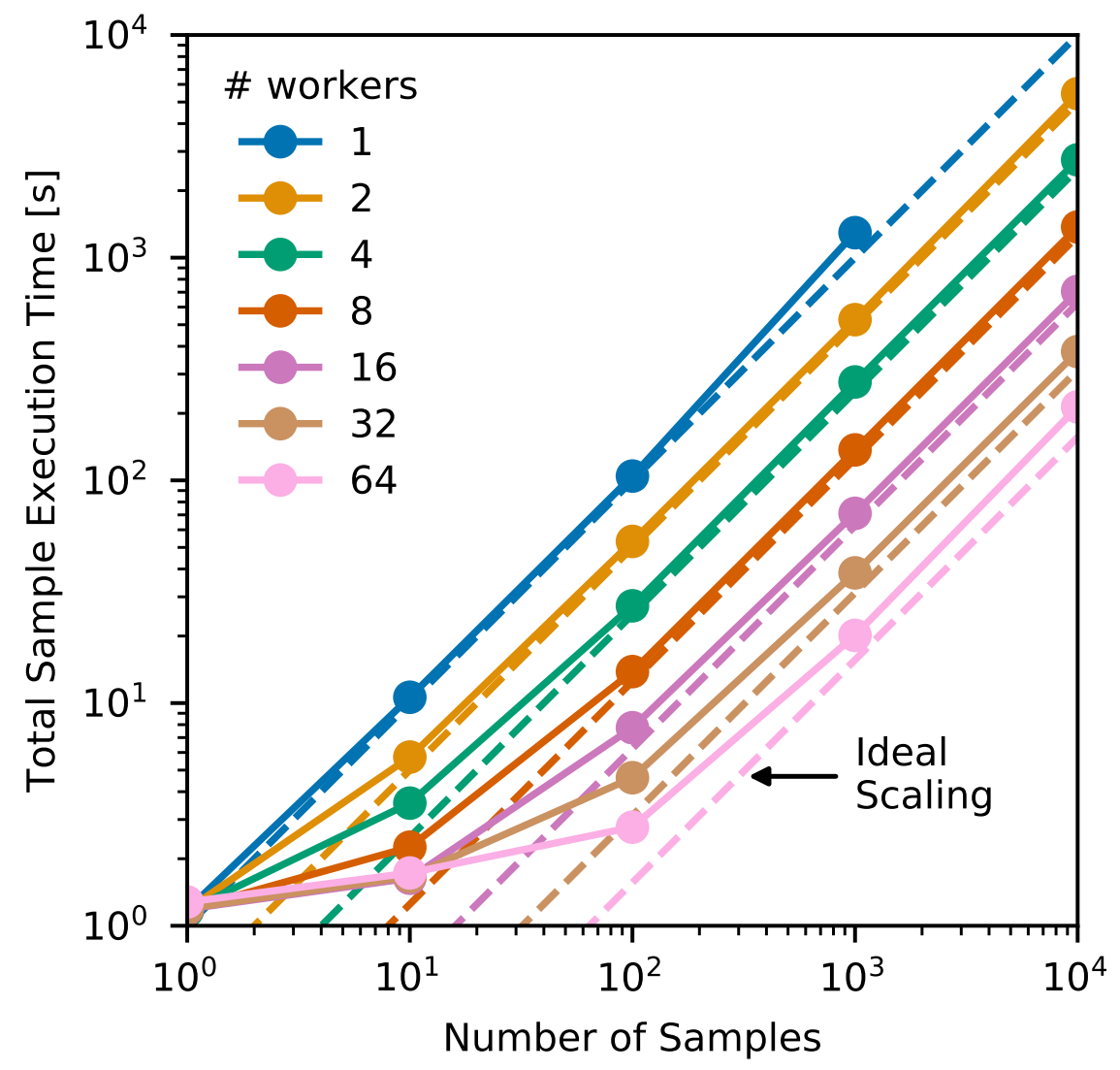

Figure 6: Sample task time. As more workers are added, heavy sample overhead is reduced. This is most easily observed at high sample volumes. 
that the overhead from ensemble generation and processing does not increase. Likewise, adding more workers has the expected scaling result of allowing the ensemble study to get executed more quickly: doubling the workers halves the time needed to process the samples.

Since the workers are decoupled from the work, Fig. 6 demonstrates that Merlin workflows can effectively take advantage of surge compute resources (just-in-time and dynamic utilization of newly available resources). As more workers come online, they can connect to the central queue server and begin processing work alongside those that are already running, without adding additional workflow overhead.

In summation, a series of null 1-second tests of varying sized ensembles quantified the overhead associated with queuing tasks, unpacking ensembles and running simulations. This overhead is small compared to what is expected for HPC-based simulations. They also showed worker scaling performs as expected. Merlin's task hierarchy algorithm allows for large ensembles to be queued and unpacked in seconds. When combined with the natural decoupling of workers from work, Merlin becomes an effective producer-consumer workflow paradigm for HPC-based simulation studies.

\section{Example Applications}

In this section, we deploy Merlin on a few real world scientific examples. We focus not on the scientific results of the studies themselves, but rather on the workflow aspects of them, how they were constructed, how they used Merlin to accomplish their goals, and what generic lessons can be learned from real world applications. Two examples are from physics, specifically inertial confinement fusion, and one is from biology, namely epidemiological studies of COVID-19 progression and mitigation. The first physics study uses JAG [22], an analytic single core python-based simulation code, and leverages the Sierra supercomputer to scale out this simple model and produce a large physics-based dataset for machine learning applications. The second physics study uses the MPI-driven multiphysics code HYDRA 23 in a machine-learning augmented optimization loop of a fusion experimental design. The COVID-19 study is a nested two-phase workflow that first calibrates an MPI agent-based epidemiological model epicast [24] and then uses the calibrated model to simulate various intervention scenarios for multiple metropolitan areas simultaneously. This set of studies collectively covers large-scale, multi-component, ML-augmented, iterative, and dynamic workflows of both MPI- and non-MPI-driven codes.

\subsection{A Scalability Study: 100 Million Fusion Simulations on Sierra}

The primary goal of Merlin is the facilitation of large-scale simulation ensembles on HPC systems. This section tests Merlin's ability to scale by showing how it created an unprecedentedly large fusion simulation dataset, consisting of the multi-variate results of roughly 100 million individual simulations on the Sierra Supercomputer at Lawrence Livermore National Laboratory. A subset of the 
data 25] has been released as an open-source dataset for the community-driven exploration of ML models suited specifically to scientific applications.

The aim of inertial confinement fusion (ICF) is to compress a hollow shell of cryogenic deuterium-tritium (DT) fuel to thermonuclear conditions. The spherical DT ice shell is encased in an ablator material, the outer surface of which is heated indirectly via x-rays generated in an encasing hohlraum (in another variant, the laser directly heats the capsule). As the capsule surface ablates, the spherical shell implodes and compresses the enclosed fuel to high temperature and density. The goal is to have the gas at the center of the shell ignite a fusion burn wave, which consumes the DT shell and releases large amounts of fusion energy. Achieving fusion ignition in the laboratory is one of the key goals of the National Ignition Facility (NIF) and would mark a major step towards the ultimate goal of clean energy from fusion-based power plants.

JAG 22] is a semi-analytic model of ICF implosions in 3D. As initial conditions, it takes two 0-dimensional physics variables and three 3-dimensional capsule perturbations and evolves an ICF capsule through the final stages of a NIF experiment. In the process it produces scalar, time-series and hyperspectral ray-traced images of the implosion, which can be directly compared to experiments. Each simulation runs in python on a single thread for approximately five minutes and outputs 48 images ( 4 frequencies $\times 3$ viewing angles $\times 4$ times), 16 time-series, 23 physics scalar quantities, 10 performance/system scalars, plus the simulation input parameters and associated meta-data.

This study used Merlin to parallelize the execution of JAG during early access time on the Sierra supercomputer. The goal was to create a large collection of simulation results, which could be used to explore the relationships between ICF observables and to train ML models for analysis of NIF experiments (see Ref. 26 for the ML research using these data). Since ML model fidelity generally improves with the data quantity, the essential aim of the study was to run JAG as many times as possible and automatically pre-process and package data for subsequent analysis. Although JAG itself does not make use of Sierra's GPUs, both Sierra's scale (no. of CPUs, size and speed of parallel filesystem) and the desire to have the dataset present on the system for GPU-driven ML applications served as motivation for using Sierra.

To maximize scalability, this study deployed a workflow (Fig. 7) that combines individual simulations into hierarchical meta-task "bundles" of tasks and data. Each bundle consisted of 10 simulations which were dumped via Conduit 21] into hdf5 files, with 100 files per leaf directory. Once each leaf directory was filled, an aggregation task collected the bundled files into a single hdf5 data file containing 1000 simulations. Each simulation produces approximately 300 $\mathrm{kB}$ of zipped hdf5 data and a 1000-simulation aggregated bundle is roughly 300 MB.

The aim was to produce $100 \mathrm{M}$ simulations using stair blue noise [27] sampling across 5 dimensions precomputed and stored in 100 independent binary files, which were read asynchronously during task creation.

The study used Flux [20 to launch Celery workers across multiple independent batch jobs of varying sizes $(64,128,256,512$, and 1024 nodes of 40 workers 


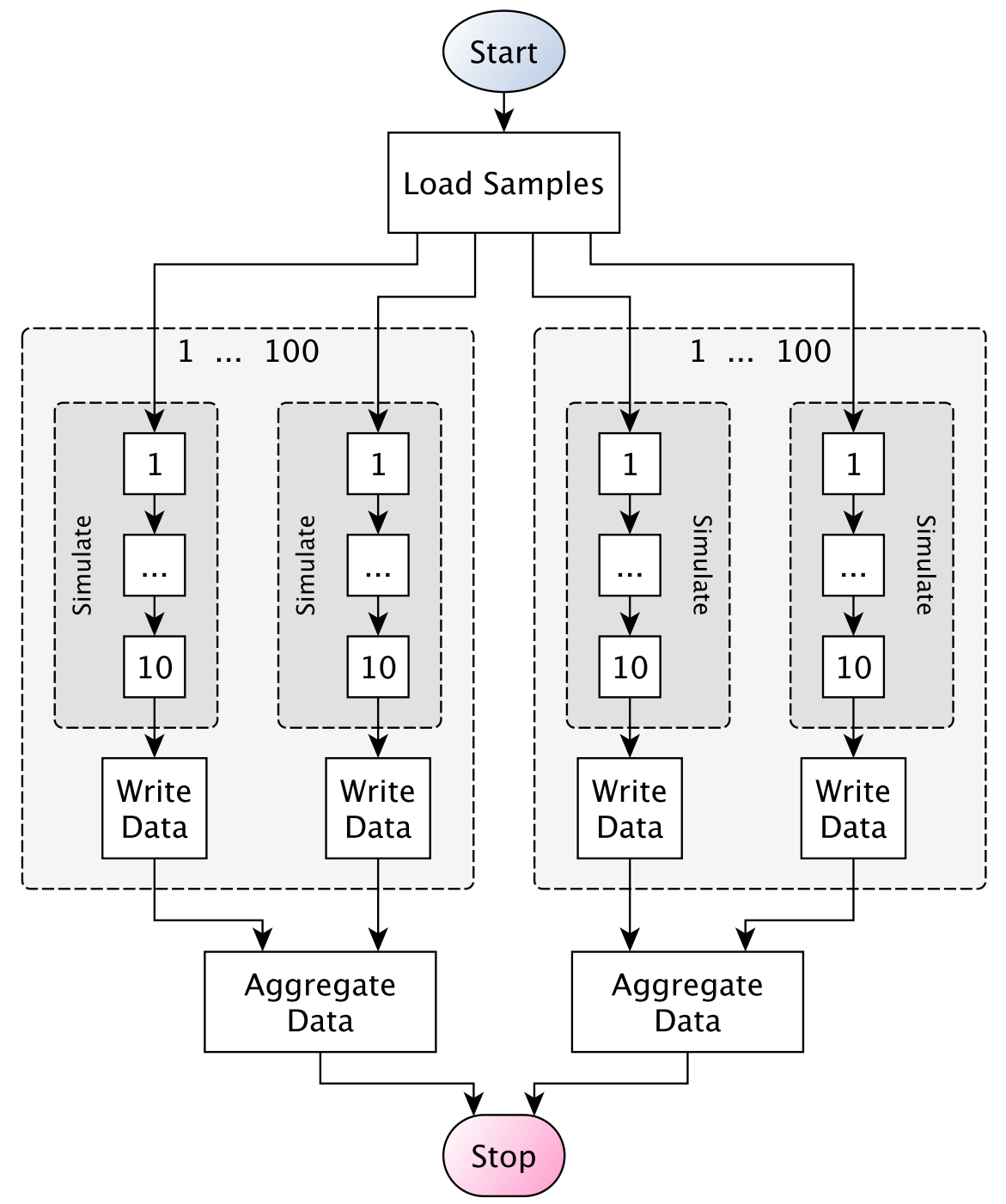

Figure 7: Hierarchical JAG workflow the leverages tasks of simulation bundles to scale production. Each task bundle consists of 10 simulations processed in serial whose results are collected in memory and then dumped to disk. Every 100 tasks, the bundled results are aggregated into files of 1000 simulations apiece. 
each, one per core) and to dynamically allocate resources as workers requested them for a new simulation bundle. Each batch job submitted itself as a dependent job via jsrun [28] to create a "worker farm" of batch requests. This scheme allowed any holes in the scheduling system to be filled with a combination of worker jobs, thereby maximizing utilization and throughput. At peak, the scheme was able to run 1024 node and 512 node jobs simultaneously, such that 61,440 concurrent workers were processing simulation requests, communicating with the Rabbit server and writing to the disk. At this rate the system was running approximately 1 million simulations and writing about $100 \mathrm{~GB}$ of physics data per wall-clock hour.

The initial run of tasks showed approximately a $70 \%$ completion rate, with the main reason for failing tasks being file system (I/O) and node failures during the volatile early access period. A second run, in which Merlin tasks first crawled the directory tree and resubmitted missing simulations back to the Rabbit task queue brought the success rate to $85 \%$. After one final pass of task resubmissions, 99755022 simulations completed successfully, with only 220978 failing due internal (physics) errors. The dataset totaled 24TB spread out across 99976 files. Its size (in number of samples and total volume) and complexity (scalars, time series, hyperspectral images) alone make it a cutting edge scientific dataset (see Sec. 1).

The study took advantage of some features of the Merlin workflow system. Firstly, the producer-consumer task queuing system naturally separated what is to be run from where it is to be run, allowing for the exploitation of multiple concurrent batch jobs of different allocation sizes. This increased system utilization and reduced batch queue wait times. Secondly, the hierarchical data directory system, the use of hdf5, and task result bundling allowed the asynchronous creation of a large dataset without the need for file locking or I/O coordination. Meta-tasks (that run multiple instances of JAG at a time) exploited on-node memory for the temporary storage of simulation results within a bundle prior to writing to disk. Thirdly, the integration with Flux for job launching enabled the just-in-time launching of tens of thousands of simultaneous simulations (at a peak rate of over $250 /$ second). This allowed the task-queue system to coordinate the sequence of work to be done without the explicit need for job and resource scheduling. Finally, the use of independent atomistic tasks created workflow resilience via a natural re-submission framework. Simulations that did not complete were easily identified through exception handling during the run, or through detection of corrupt data after-the-fact, and resubmitted to the task queue system. The decoupling of work from compute resources meant that workflow "cleanup" could happen at a later time with a smaller allocation.

\subsection{An Iterative Workflow Study: Optimizing the Design of a Fusion Experi- ment}

The example in this section tests Merlin's ability to perform iterative simulation studies of MPI-driven codes. This workflow archetype, which consists of running a set of simulations, processing the results, performing analysis and then choosing a new set of simulations is a cornerstone of scientific computing. 
It encompasses such aims as simulation-driven design and optimization, active learning, and experimental analysis. In this section, we apply Merlin to the machine-learning enhanced optimization of a nuclear fusion experiment.

A major challenge of NIF experiments is that of manufacturability precision. In particular, the target capsule and laser system are designed and optimized together in silico, such that the requested laser pulse and requested capsule geometry are meant to be perfectly matched. However, due to finite experimental precision (for instance in the delivery of the laser power source or capsule manufacturability tolerances), the as-delivered laser pulse and capsule are not as intended, nor are they guaranteed to be optimally combined.

The study [29] discussed in this section attempts to re-optimize the target capsule to align with the delivered laser pulse of a particular experiment and in so doing to take into consideration expected levels of capsule manufacturability variations. In other words, the goal is to maximize the expected nuclear yield under random draws from a prescribed capsule geometry. This problem setup necessitates constructing an optimization cost function that for each design point considers a number of small variations about that design point. Furthermore, the problem is one of constrained optimization that can consider expert judgment for "valid" regions of parameter space: it is not sufficient to merely maximize yield, but rather to maximize yield subject to other simulation outputs (for instance, such that the capsule implosion velocity remain below a threshold, above which the experiment is unlikely to behave as predicted by simulations). Additionally, ICF capsule geometries can have 5-15 design parameters, which means that an iterative approach is likely to be more successful than a single-iteration approach (e.g. as done in Ref. [2, which optimized a ML model built on 60,000 simulations in 9 dimensions). Another challenge is that the multiphysics design code, HYDRA, can be of moderate computational cost (if run in 1D), taking approximately 10-15 minutes on one core. This makes it too costly to run directly when calculating the objective function, but quick enough to where workflow overhead could become a significant fraction of compute time. Calculating the physics variables that feed into the objectives and constraints requires post-processing of simulation data (both raw data and intermediate data calculated in situ), which means that the vast majority of data produced is of little value for optimization, but might be of interest for subsequent analysis of any resulting design.

Fig. 8 shows the workflow used to re-optimize the NIF ICF target capsules under uncertainties and constraints. The workflow leverages the producerconsumer model to perform a number of iterative loops. During each iteration, workers running under Flux launch and post-process simulations asynchronously. After a simulation is done post-processing, a worker deletes its raw data to save space and inode counts. Once all of the post-processing is completed, another task collects the data and extracts those features relevant for optimization. Then, a worker trains machine learning surrogate models for those features extracted from all previous iterations and saves them to disk. Subsequently, an optimization task loads those surrogate models and feeds them into an optimization algorithm that queries the models several times at each design 


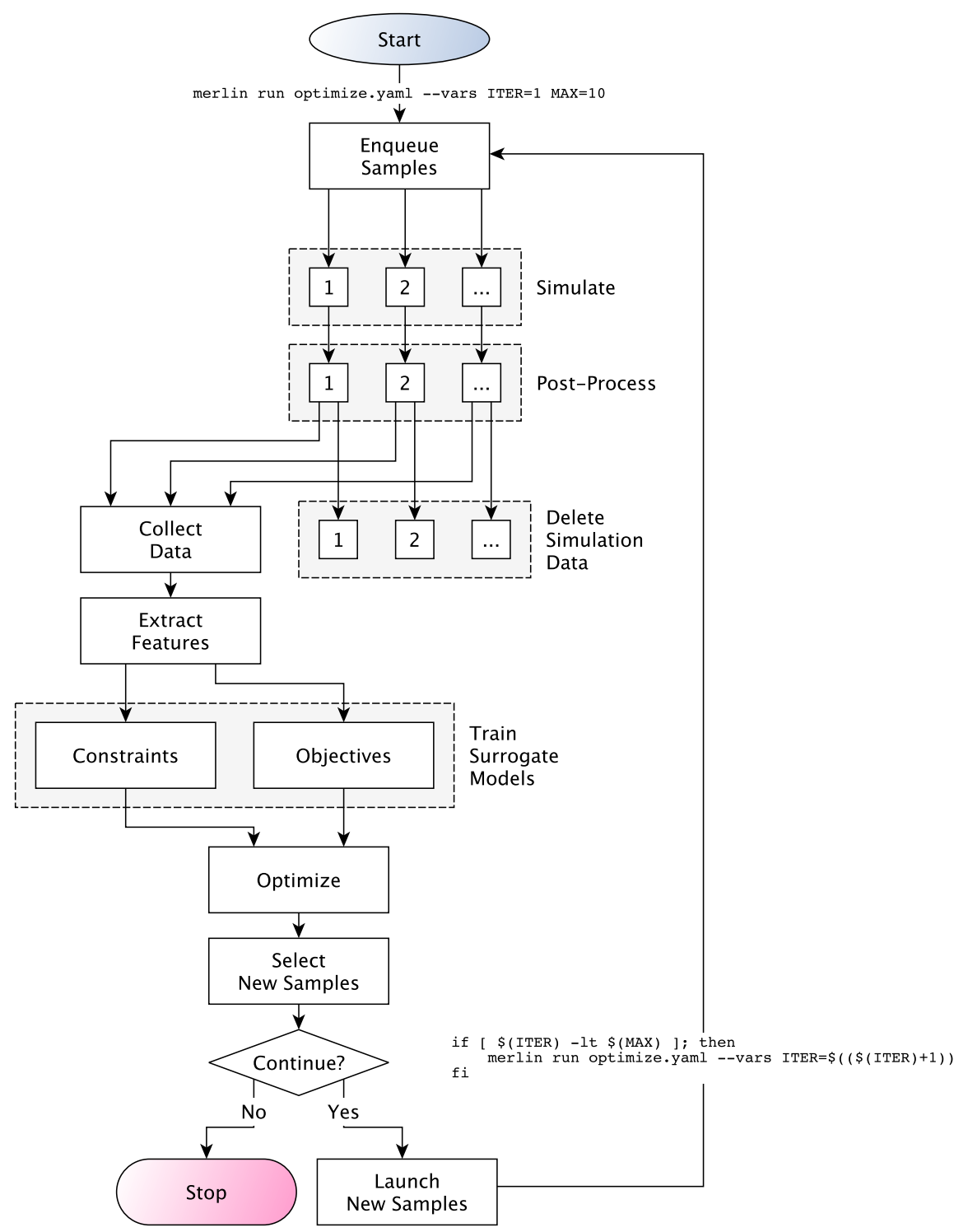

Figure 8: Iterative workflow for surrogate-augmented optimization of fusion experiments. The optimization chain is initiated with a single call to Merlin that sets the maximum number of allowed iterations. Subsequent iterations are automatically called with a worker task call that uses bash syntax to determine if another iteration should be launched. 
point to produce both the cost function and evaluate the constraints. Once the surrogates have been optimized, a new search area is defined and new simulation samples chosen. Finally, the new samples are re-queued with a final worker call to Merlin that also increments a variable defined in the workflow file to keep track of the iteration count.

The workflow was tested on the Lassen supercomputer, with 5 independent 2-node jobs of workers. Like the "worker farm" concept of the JAG study, each job resubmitted a dependent child job. With 80 total worker threads per job, the scheme allowed for up to 400 concurrent workers when all jobs were running at the same time. However, at any given time any of the 5 of the batch jobs could be executing and contributing to the study. Each iteration produced 384 new simulations (128 around the best existing point, 128 at the predicted optimal and 128 connecting the two regions). To avoid wasting resources (for instance, by idling working while waiting for a single iteration to complete or while waiting for the last post-process task to complete prior to the synced collection step), multiple studies were launched at once under different constraint conditions, so that there was always work queued. This means that at any given time, workers on the same batch allocation were potentially working on different problems. Since both the re-queuing of simulation iterations and the resubmitting of workers to the batch system were automatic, the process was largely hands-off and produced roughly 100,000 simulations over the course of a few days calendar time (25,000 CPU-hours for the simulations spread out among multiple workers and batch allocations). A few optimization chains had to be manually resubmitted when system failures caused a particular chain to stop, much like during the 100M JAG study. But since the work is decoupled from the workers, this could be done without pausing or relaunching batch job allocations.

The optimization study benefited from a few features of Merlin. Firstly, the decoupling of producers and consumers meant that iterations could be automatic and computer resources used to work on multiple iterations and problems simultaneously without having to wait for dedicated batch jobs. It also allowed optimization chains that failed due to system issues to be resubmitted in real time. The shell-like API allowed for flow-control logic and the iterative and dynamic launching of chains, as well as the stitching of MPI-driven codes with post-processing, optimization and machine learning tools, and the easy integration with Flux for on-demand resource allocation (e.g. combining multiple instances of HYDRA onto a single shared node).

\subsection{A Mixed Parameter-Sample Nested Workflow Study: Simulating COVID- 19 Intervention Scenarios}

In this section, we describe a study that takes advantage of Merlin's lowoverhead HPC-based producer-consumer workflow model to create a cascading workflow. The goal of this work [30, 31] was to explore possible nonpharmaceutical intervention scenarios to slow the advance of the COVID-19 pandemic with an agent-based epidemiological model, epicast [24]. epicast is a large-scale parallel MPI-based model of infectious diseases originally built to 
model influenza. As an existing tool, epicast was attractive for its potential use in the triage of possible intervention measures (e.g. whether to close schools or restaurants), but it (obviously) hadn't been applied to COVID-19 before. Doing so came with a number of challenges that Merlin helped overcome.

epicast uses the latest census data to create a virtual population of agents that mimic population characteristics, such as age distribution, household size, job type, commuting patters, etc., on a census tract level (roughly 2000 people), which are subsequently combined to approximate counties, metropolitan areas, states, and even the entire nation. Unsurprisingly, the model depends on a number of parameters describing the initial conditions of an outbreak, disease biology, and social variables like the expected compliance with social distancing. Some of these parameters are "global" in that they are similar in different locations (e.g. the innate infectivity of a particular disease), while some are "local" in that they could be unique to a particular geographical region. In order to effectively use epicast to either forecast potential outcomes or explore mitigation scenarios, all of these parameters must be first accurately calibrated.

Essentially, early in the outbreak, epicast researchers needed to quickly find new valid parameter sets and then use these sets to launch more simulations of possible projection scenarios. With ground-conditions frequently changing and uncertain, these parameters were unknown. The research need therefore was the ability to run large quantities of small-scale simulations with low-overhead (epicast itself could simulate a single metropolitan region under one scenario/parameter set on 64 cores of one Knights Landing (KNL) node in approximately 3 minutes) to find likely parameter sets valid for the disease and then to follow up each set with a series of simulations projecting forward in time and studying the effects of different possible intervention scenarios. Finally there was a need to organize and automate the distinction between "global" and "local" parameter sets and fold in post-processing and data packaging for later analysis. With the time pressures inherent in the outbreak, epicast researchers could not spend large quantities of time learning new workflow APIs and wanted to be able to exploit large computational resources made available to the problem across multiple supercomputer sites.

Figure 9 shows a diagram of the two-step cascading workflow for the study of COVID-19 interventions. It defines DAG parameter sets per geographic region and reserves samples for disease conditions and intervention strategies. The first phase of the workflow ("calibration") pulls the latest COVID-19 case data and uses Flux to launch a series of "pre-ensembles" to initialize the simulation code under different ground conditions and model parameters. At the end of the workflow, it refines its estimate of these parameters to match the data and then launches the second phase, which consists of longer running forecasting models under different intervention scenarios, followed by traditional data processing and packaging for subsequent analysis.

Merlin provided a few enabling features for this study. Firstly, since worker steps can issue calls to merlin run and since this operation is very fast (see Fig. 3), the two-step workflow could be automatic and dynamic. Secondly, the parameter-sample expansion distinction allowed for the asynchronous parallel 


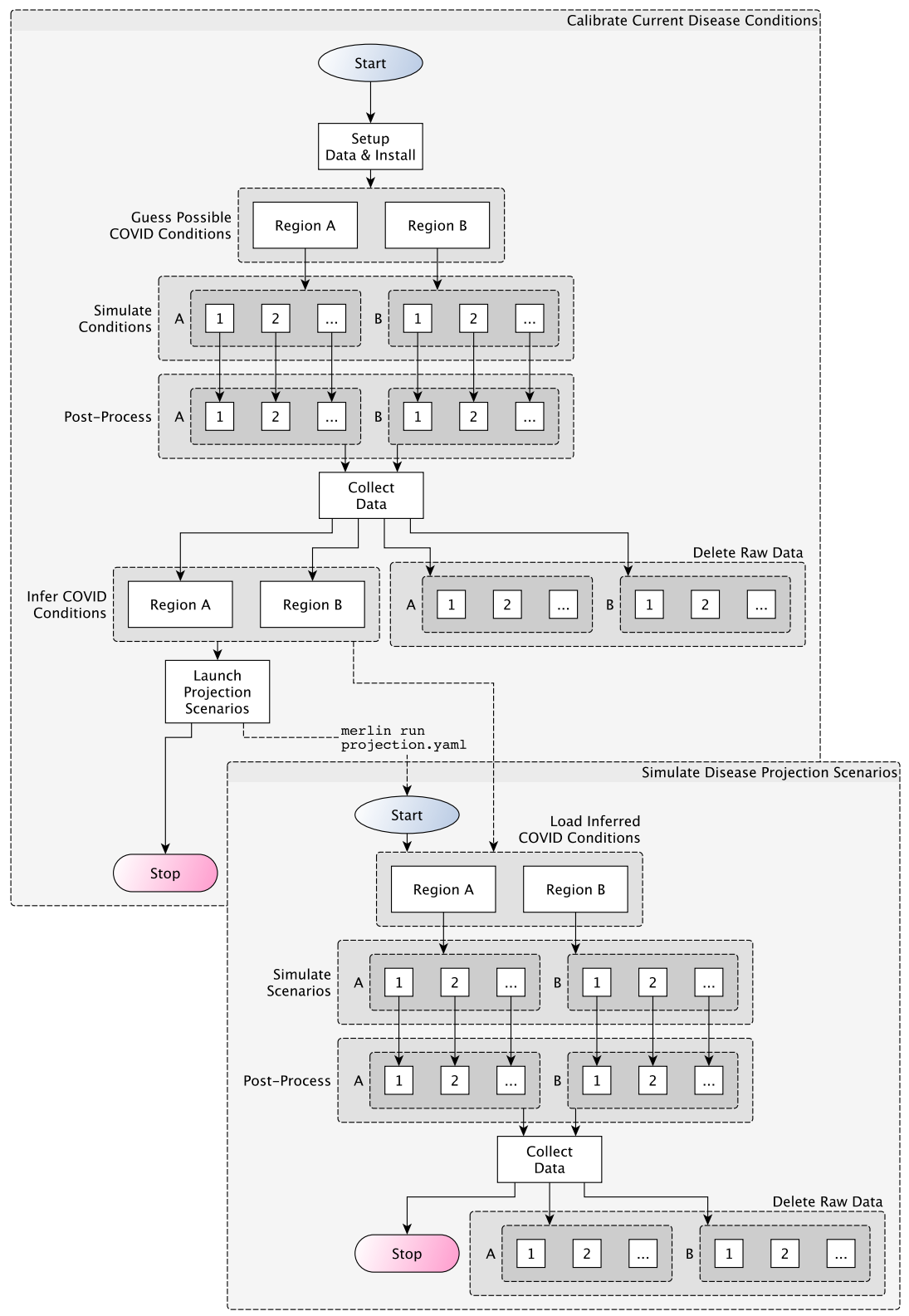

Figure 9: Cascading workflow for studying the efficacy of non-pharmaceutical interventions for COVID-19. The first phase runs a series of agent-based epidemiological simulations to calibrate model parameters to fit current disease conditions and then launches the second phase, which simulates possible intervention scenarios. 
calibration and study of multiple metropolitan areas. Thirdly the low workflow overhead per task meant that few resources were spent to workflow management, as compared to simulation compute. Fourth, the producer-consumer model meant that existing and surge-capacity workers could process many different scenarios without waiting for new batch allocations. Since the workers are decoupled from the work queue, multiple machines from the same compute center could be stitched together to form a federated system and increase throughput. Finally, by building upon existing open-source technology, Merlin was able to be deployed at multiple supercomputing sites, allowing significant computational resources to be allocated to the problem, while the shell-centered API allowed for the execution of the custom libraries needed to run the underlying code through Flux at each site.

\subsection{Conclusions from Real-World Examples}

This section presented three real world examples of Merlin's use for scientific studies. Together, some common key results and lessons emerge from Merlin's application.

Firstly, it's possible to use Merlin to run large scale real world simulation ensemble studies. Good scalability from the JAG study came in large part to the ability to leverage Conduit/hdf5 for on-the-fly data bundling and aggregation. All studies used Flux to efficiently allocate resources when needed. The optimization study made judicious use of in-transit data deletion to save disk space. For these quickly running simulations, the relatively low overhead for a Merlin task meant that compute resources could be devoted primarily to simulations. Another key to maximizing throughput in large-scale studies was the decoupling of producers and consumers, with separate batch allocations dedicated to running generic worker tasks that could pull work from shared queues. Not only did the shell-based API allow for multiple codes to be stitched together into complicated workflows by non-experts, but it also allowed for the flexibility needed to run simulations at HPC centers: both epicast and HYDRA required machine-specific special libraries to be linked and runtime environment variables set to properly run under Flux. The use of a shell-API combined with a producer-consumer model also allowed for workflows to break free of static DAG constructions to incorporate such constructs as looping and branching. Finally the distinction and separation between DAG-based parameters (with possibly complex dependencies) and samples (with simple dependencies but potentially large numbers) allows for both scalability in task creation and flexibility in workflow definition.

In two of the studies, system errors caused some jobs to fail, so the ability to resubmit tasks to the queue system without interrupting running workers helped minimize time spent waiting in a batch queue scheduler before correcting results, allowing resources to pick up naturally where the study left off.

The major challenge for studies using Merlin in all cases was interfacing with third party libraries. In particular, although Flux and Conduit are not software dependencies, using them for their scalability benefits means that they must be installed and working separately at an HPC center. Furthermore, a 
Celery-supported queue server and backend (eg. RabbitMQ and Redis) also need to be deployed and instantiated on dedicated resources for workers to coordinate across batch allocations. Recent advances in container technology are making this installation easier, and such a strategy was used to deploy Merlin at Lawrence Berkeley National Lab/NERSC and Oak Ridge National Laboratory for the COVID-19 study (using spin [32] at NERSC and slate/openshift [33] at Oak Ridge). The biggest challenge to using Merlin is the one-time up-front investment in the architecture supporting cloud-based technologies. However, as compute centers move to incorporate more of these data services into their portfolios, porting Merlin to new locations should become easier. It may also be possible to explore containerized solutions on compute nodes within a batch allocation, establishing virtual pop-up servers, but creating a robust solution accessible to multiple nodes across batch allocations would be an open area of research.

\section{Conclusion}

Merlin is a workflow framework designed to facilitate the creation of large scale ensembles of HPC-driven simulations. With an eye toward future integrated ML-driven workflows, it combines distributed asynchronous task-queuing software with HPC technologies and allows for the integration with flexible hierarchical data formats and next-generation batch schedulers. Merlin's shell-like intuitive interface provides the flexibility required to execute MPI-driven multiphysics simulations in a leadership-class HPC environment.

In this paper we presented Merlin's motivation and design, tested its performance and scalability on an idealized workflow, and demonstrated its use on three real world scientific problems. The idealized test problem showed Merlin's capacity to scale workflow ensembles to several million samples with low overhead, empowered by Merlin's dynamic hierarchical task-generation algorithm. Through the 100M JAG dataset, we demonstrated how Merlin can scale to thousands of nodes and coordinate tens of thousands of asynchronous workers. When combined with Flux and deployed on Sierra, it was able to process roughly 1 million ICF simulations per hour to create a uniquely rich massive simulation ensemble dataset, which because of its size and complexity, could serve as a benchmark to stress advanced ML systems. The ICF optimization problem leveraged Merlin's consumer-producer model and low task queuing overhead to create a dynamic workflow that uses ML to steer the optimization of an MPIdriven code. Finally the epicast COVID-19 study built a dynamic workflow that exploited the separation of parameters and samples that was both intu-

itive and scalable. Furthermore, the COVID-19 work was able to use Merlin's producer-consumer model to stitch together multiple HPC systems within a compute center into a coordinated resource, thereby enabling greater throughput of time-sensitive results. All examples benefited from surge computing, low task generation and execution overhead, and the shell-like API. Finally, all examples experienced random system failures that prevented some tasks from 
succeeding. Nonetheless, Merlin's provenance and resubmission framework allowed the users to recover from the failures in real time or after the fact, without having to wait for new dedicated compute resources.

Merlin has been deployed at multiple HPC centers and is built using opensource components. Both Merlin ${ }^{2}$ and a 10,000-sample subset of the JAG dataset $t^{3}$ are available publicly. The merlin spellbook ${ }^{7}$ is a companion project that contains useful routines and components for building workflows, such as sample generation, building ML surrogate models and data packaging.

Future work includes developing an API for periodic tasks and tools to facilitate job monitoring, clean-up, and resubmission. Some examples come packaged with Merlin (including the null example used to test performance), but additional templated workflows and worker submission scripts could allow for increased user adoption. Finally it may be possible to write Merlin interfaces to other workflow descriptions beyond the Maestro YAML file.

While Merlin itself pushes the boundaries of contemporary simulation ensemble creation, our work demonstrates above all that a confluence of distributed and traditional HPC technologies represents a promising path towards the realization of next-generation ML-integrated scientific computing.

\section{Acknowledgment}

The authors wish to thank the Flux and Conduit developers, as well as the computing centers at LLNL, LBNL, and ORNL.

This work was performed under the auspices of the U.S. Department of Energy by Lawrence Livermore National Laboratory under contract DE-AC5207NA27344. Lawrence Livermore National Security, LLC. This document was prepared as an account of work sponsored by an agency of the United States government. Neither the United States government nor Lawrence Livermore National Security, LLC, nor any of their employees makes any warranty, expressed or implied, or assumes any legal liability or responsibility for the accuracy, completeness, or usefulness of any information, apparatus, product, or process disclosed, or represents that its use would not infringe privately owned rights. Reference herein to any specific commercial product, process, or service by trade name, trademark, manufacturer, or otherwise does not necessarily constitute or imply its endorsement, recommendation, or favoring by the United States government or Lawrence Livermore National Security, LLC. The views and opinions of authors expressed herein do not necessarily state or reflect those of the United States government or Lawrence Livermore National Security, LLC, and shall not be used for advertising or product endorsement purposes.

Released as LLNL-JRNL-821884.

\footnotetext{
${ }^{2}$ Merlin is available under the MIT license: https://github.com/LLNL/merlin

${ }^{3}$ The JAG dataset is available via the LLNL Open Data Initiative: https://data-science.llnl.gov/open-data-initiative

${ }^{4}$ The merlin spellbook collection of workflow components is available at https://github.com/LLNL/merlin-spellbook.
} 


\section{References}

[1] E. I. Moses, R. N. Boyd, B. A. Remington, C. J. Keane, R. Al-Ayat, The national ignition facility: Ushering in a new age for high energy density science, Phys. Plasmas 16 (041006) (2009) 1-13.

[2] J. L. Peterson, K. D. Humbird, J. E. Field, S. T. Brandon, S. H. Langer, R. C. Nora, B. K. Spears, P. T. Springer, Zonal Flow Generation in Inertial Confinement Fusion Implosions, Physics of Plasmas 24 (3) (2017) 032702. doi:10.1063/1.4977912,

[3] D. Dua, C. Graff, UCI machine learning repository, http://archive.ics. uci.edu/ml (2017).

[4] P. Schmidt, A. Reiss, R. Duerichen, C. Marberger, K. Van Laerhoven, Introducing wesad, a multimodal dataset for wearable stress and affect detection, in: ACM Int. Conf. on Multimodal Interaction, ICMI '18, 2018, pp. $400-408$.

[5] P. Baldi, P. Sadowski, D. Whiteson, Searching for exotic particles in highenergy physics with deep learning, Nature Communications 5 (2014) 4308 $\mathrm{EP}-$.

[6] T. Miyoshi, K. Kondo, K. Terasaki, Big Ensemble Data Assimilation in Numerical Weather Prediction, Computer 48 (11) (2015) 15-21.

[7] S. H. Langer, B. Spears, J. L. Peterson, J. E. Field, R. Nora, S. Brandon, A HYDRA UQ Workflow for NIF Ignition Experiments, in: Workshop on In Situ Infrastructures for Enabling Extreme-Scale Analysis and Visualization, 2016, pp. 1-6.

[8] E. Deelman, K. Vahi, G. Juve, M. Rynge, S. Callaghan, P. J. Maechling, R. Mayani, W. Chen, R. Ferreira da Silva, M. Livny, K. Wenger, Pegasus: a Workflow Management System for Science Automation, Future Gener. Comput. Syst. 46 (2015) 17-35.

[9] A. Jain, S. P. Ong, W. Chen, B. Medasani, X. Qu, M. Kocher, M. Brafman, G. Petretto, G.-M. Rignanese, G. Hautier, D. Gunter, K. A. Persson, FireWorks: a Dynamic Workflow System Designed for High-throughput Applications, Concurrency and Computation: Practice and Experience 27 (17) (2015) 5037-5059.

[10] E. J. Friedman-Hill, E. L. Hoffman, M. J. Gibson, R. L. Clay, K. H. Olson, Incorporating Workflow for V\&V/UQ in the Sandia Analysis Workbench., Tech. rep., Sandia National Lab. (2015).

[11] T. Dahlgren, D. Domyancic, S. Brandon, T. Gamblin, J. Gyllenhaal, R. Nimmakayala, R. Klein, Scaling Uncertainty Quantification Studies to Millions of Jobs, in: Proceedings of the 27th ACM/IEEE International Conference for High Performance Computing and Communications Conference (SC), 2015. 
[12] J. Gyllenhaal, T. Gamblin, A. Bertsch, R. Musselman, Enabling High Job Throughput for Uncertainty Quantification on BG/Q, in: IBM HPC Syst. Sci. Comput. User Group (ScicomP'14), 2014.

[13] M. Wilde, M. Hategan, J. M. Wozniak, B. Clifford, D. S. Katz, I. Foster, Swift: A Language for Distributed Parallel Scripting, Parallel Comput. 37 (9) (2011) 633-652.

[14] I. Foster, M. Ainsworth, B. Allen, J. Bessac, F. Cappello, J. Y. Choi, E. Constantinescu, P. E. Davis, S. Di, W. Di, H. Guo, S. Klasky, K. K. Van Dam, T. Kurc, Q. Liu, A. Malik, K. Mehta, K. Mueller, T. Munson, G. Ostouchov, M. Parashar, T. Peterka, L. Pouchard, D. Tao, O. Tugluk, S. Wild, M. Wolf, J. M. Wozniak, W. Xu, S. Yoo, Computing Just What You Need: Online Data Analysis and Reduction at Extreme Scales, in: Euro-Par 2017: Parallel Processing, 2017, pp. 3-19.

[15] J. Ozik, N. T. Collier, J. M. Wozniak, C. Spagnuolo, From Desktop to Large-Scale Model Exploration with Swift/T, in: Winter Simul. Conf., 2016, pp. 206-220.

[16] F. Di Natale, Maestro Workflow Conductor, https://github.com/LLNL/ maestrowf (Mar. 2019).

[17] Celery: Distributed Task Queue, http://www.celeryproject.org/ (2019).

[18] M. Rostanski, K. Grochla, A. Seman, Evaluation of highly available and fault-tolerant middleware clustered architectures using rabbitmq, in: Federated Conf. on Comput. Sci. and Inf. Syst., 2014, pp. 879-884.

[19] J. Han, E. Haihong, G. Le, J. Du, Survey on nosql database, in: IEEE Int. Conf. on Pervasive Comput. and Appl., 2011, pp. 363-366.

[20] D. H. Ahn, N. Bass, A. Chu, J. Garlick, M. Grondona, S. Herbein, J. Koning, T. Patki, T. R. Scogland, B. Springmeyer, et al., Flux: Overcoming Scheduling Challenges for Exascale Workflows, in: IEEE/ACM Workflows in Support of Large-Scale Science (WORKS), 2018, pp. 10-19.

[21] C. Harrison, et al., Conduit: Simplified Data Exchange for HPC Simulations, https://software.llnl.gov/conduit (Mar 2019).

[22] J. Gaffney, D. Casey, D. Callahan, E. Hartouni, T. Ma, B. Spears, Data Driven Models of the Performance and Repeatability of NIF High Foot Implosions, in: APS Meeting Abstracts, 2015.

[23] M. M. Marinak, G. D. Kerbel, N. A. Gentile, O. Jones, D. Munro, S. Pollaine, T. R. Dittrich, S. W. Haan, Three-dimensional HYDRA simulations of National Ignition Facility targets, Physics of Plasmas 8 (4) (2001) 22755. 
[24] P. Fenimore, B. McMahon, N. Hengartner, T. Germann, J. Mourant, A suite of mechanistic epidemiological decision support tools, Online Journal of Public Health Informatics 10 (1) (2018) e1.

[25] R. Anirudh, JAG ICF Dataset for Scientific Machine Learning (Oct. 2019). URL https://github.com/rushilanirudh/icf-jag-cycleGAN

[26] R. Anirudh, J. J. Thiagarajan, P.-T. Bremer, B. K. Spears, Improved surrogates in inertial confinement fusion with manifold and cycle consistencies, Proceedings of the National Academy of Sciences 117 (18) (2020) 97419746. doi:10.1073/pnas.1916634117.

URL https://www . pnas .org/content/117/18/9741

[27] B. Kailkhura, J. J. Thiagarajan, P.-T. Bremer, P. K. Varshney, Stair Blue Noise Sampling, ACM Trans. Graph. 35 (6) (2016) 248:1-248:10.

[28] IBM Knowledge Center, jsrun, https://www.ibm.com/support/ knowledgecenter/SSWRJV_10.1.0/jsm/jsrun.html (2021).

[29] L. Peterson, B. Kustowski, L. Masse, J. Koning, B. Bay, J. Gaffney, K. Humbird, M. Kruse, R. Nora, B. Spears, Engineering robustness into inertial confinement fusion designs, Bulletin of the American Physical Society.

[30] R. Anirudh, J. J. Thiagarajan, P.-T. Bremer, T. C. Germann, S. Y. D. Valle, F. H. Streitz, Accurate calibration of agent-based epidemiological models with neural network surrogates (2020). arXiv:2010.06558.

[31] J. J. Thiagarajan, P.-T. Bremer, R. Anirudh, T. C. Germann, S. Y. D. Valle, F. H. Streitz, Machine learning-powered mitigation policy optimization in epidemiological models (2020). arXiv:2010.08478.

[32] NERSC, Spin (Dec. 2020).

URL https://www.nersc.gov/systems/spin/

[33] G. Papadimitriou, K. Vahi, J. Kincl, V. Anantharaj, E. Deelman, J. Wells, Workflow submit nodes as a service on leadership class systems, in: Practice and Experience in Advanced Research Computing, PEARC '20, Association for Computing Machinery, New York, NY, USA, 2020, pp. 56-63. doi:10.1145/3311790.3396671.

URL https://doi.org/10.1145/3311790.3396671 